\title{
Combfit: A Normalization Method for Array CGH Data
}

\author{
Shigeyuki Oba, ${ }^{\dagger}$ Nobumoto Tomioka,${ }^{\dagger \dagger}$ Miki Ohira ${ }^{\dagger \dagger}$ \\ and SHIN ISHII ${ }^{\dagger}$
}

\begin{abstract}
The recently developed array-based comparative genomic hybridization (array CGH) technique measures DNA copy number aberrations that occur as causes or consequences of cell diseases such as cancers. Conventional array CGH analysis classifies DNA copy number aberrations into three categories: no significant change, significant gain, and significant loss. However, recent improvements in microarray measurement precision enable more quantitative analysis of copy number aberrations. We propose a method, called comb fitting, that extracts a quantitative interpretation from array CGH data. We also propose modifications that allow us to apply comb fitting to cases featuring heterogeneity of local aberrations in DNA copy numbers. By using comb fitting, we can correct the baseline of the fluorescence ratio data measured by array CGH and simultaneously translate them into the amount of changed copy numbers for each small part of the chromosome, such as $0, \pm 1, \pm 2, \cdots$. Comb fitting is applicable even when a considerable amount of contamination by normal cells exists and when heterogeneity in the ploidy number cannot be neglected.
\end{abstract}

\section{Introduction}

The recently developed array-based comparative genomic hybridization (array CGH) technique measures DNA copy number aberrations that occur as causes or consequences of cell diseases such as cancers ${ }^{1), 2), 6), 11), 14) ~ 18)}$. The segmentation structure of chromosomal aberrations is of major interest because segmental gains or losses often cause or reflect cell diseases. Fridlyand, et al. $(2004)^{5)}$ assumed that measured copy number aberrations could be generated by a hidden Markov model (HMM) with latent segmentation structures, which was estimated by a forward-backward algorithm. Daruwala, et al. $(2004)^{3)}$ proposed a similar model to the HMM and calculated the optimum segmentation structure by a dynamic programming algorithm. There has been a great deal of other research on the segmentation problem such as ${ }^{8), 9), 12), 13)}$. Such sequential segmental structures are also used for noise reduction, and there exist many approaches other than segmentation, such as simple moving average ${ }^{2)}$, penalized quantile smoothing ${ }^{4)}$, and wavelet filter $^{7)}$. For segmentation and noise reduction, other research efforts have compared alternative methods $\left.{ }^{10)}, 19\right)$.

$\dagger$ Graduate School of Information Science, Nara Institute of Science and Technology

$\dagger \dagger$ The 1st Department of Surgery, Hokkaido University, Graduate School of Medicine

$\dagger \dagger \dagger$ Division of Biochemistry, Chiba Cancer Center Research Institute
Assigning an appropriate copy number of DNA is the next important issue, since what we can directly observe is the fluorescent level of each spot corresponding to each BAC (bacterial artificial chromosome) clone that is complementary to the objective piece of the sample DNA. Furthermore, the fluorescent level inevitably includes biases and variances from various causes. In many previous studies, copy number aberrations of DNA were classified into categories of no significant change, significant loss, significant gain, and sometimes large amplification. In this study, we present a method that extracts quantitative interpretation from array $\mathrm{CGH}$ data, called comb fitting. Using this method, we can correct the baseline of the fluorescence ratio data for each clone of each sample measured by array CGH and simultaneously transform the data into a numerical copy number for the changes of clones, such as $0, \pm 1, \pm 2, \cdots$. Consequently, this improves the analysis of phenomena observed on chromosomes.

\section{Formal Description on Chromoso- mal Aberrations}

Each chromosome in somatic cells normally has two DNA copies, and chromosomal aberration in cancer cells sometimes causes aneuploidy, i.e. the total copy number becomes three, four, five or more; these are called triploid, tetraploid, pentaploid, and so on. These ploidy numbers are described as $N_{\mathrm{P}}=$ $2,3,4,5, \cdots$. 
Various other types of copy number aberrations can be mentioned:

- Copy number gains in the whole or a part of a chromosome, which correspond to several or more $\mathrm{BAC}$ clones. We call these +1 gain, +2 gain, and the like.

- Copy number losses in the whole or a part of a chromosome, which correspond to several or more BAC clones. We call these +1 loss, +2 loss, and the like.

- Copy number gain whose amount is usually larger than ten, in a small part of a chromosome; we call this an amplification.

Each of these events is generally called a local aberration, which denotes the number of local gains or losses of copies and is expressed by $N_{\mathrm{C}}$.

First, all cancer cells in an objective sample are assumed to have homogeneous genetic aberrations. $N_{i j}$ denotes a copy number of the $i$ th piece of chromosome corresponding to the $i$ th $\mathrm{BAC}$ clone in the $j$ th sample; in the following, we call it simply a copy number of the $i$ th clone. The copy number is an integer, $N_{i j} \in$ $\{0,1,2, \cdots\}$, and is the sum of the ploidy number and the local aberration, $N_{i j}=N_{\mathrm{P} j}+N_{\mathrm{C} i j}$. In the conventional array $\mathrm{CGH}$ analysis, we are interested in local aberration, $N_{\mathrm{C}}$, rather than $N_{\mathrm{P}}$, because $N_{\mathrm{P}}$ can be observed by other conventional methods, and the loci and amounts of local aberrations are believed to have important information about the characteristics of the cancer. The actual measurement involves some degree of noise; therefore, obtaining the expected local aberration, $Z$, which is called the mean local aberration, is the major aim of our method.

Next, we consider the heterogeneity of the cell characters in an objective sample, which are due to the various reasons listed below.

- (a) Contamination by normal cells

- (b) Presence of multiple types of chromosomal aberration

- 1. heterogeneity in ploidy number

- 2. heterogeneity in local aberrations

This heterogeneity presents many difficulties for quantitative analyses of chromosomal aberrations.

If the amount of contamination by normal cells is known, in case (a), then we can correct these effects by using the knowledge that $N_{\mathrm{P}}=2$ and $N_{\mathrm{C}}=0$ in normal cells. When we do not know the amount of contamination, however, the correction is based on an estimation; this estimation is also provided in our frame- work (see Section 3.3).

In case (b).1, we need another correction that depends on the mixing ratios of ploidy numbers, but this can be made similarly to case (a) (see Section 3.3).

In case (b).2, $Z$ is regarded as the mean of $N_{\mathrm{C}}$ over the cells in a sample; we call this the mean local aberration. For example, when a tumor sample consists of the same amount of cells with $N_{\mathrm{C}}=-1$ and $N_{\mathrm{C}}=-2$, the mean local aberration becomes -1.5. Our method intends to obtain the real number $Z_{i j}$ rather than the integer $N_{\mathrm{C} i j}$.

When sample cells are sufficiently homogeneous with respect to $N_{\mathrm{C}}$ values, the true mean local aberration $Z$ is an integer for almost every BAC clone. Consequently, when $Z$ is estimated as, for example, $Z=1.1$, we assume that the real $Z$ is +1.0 , including a noise contribution of 0.1 . Note, however, that we cannot eliminate from this observation the possibility of a mixture containing $90 \% N_{\mathrm{C}}=1$ and $10 \% N_{\mathrm{C}}=2$.

Microarray technology measures fluorescence levels of CY3 $\left(\mathrm{CY} 3_{i j}\right)$ and CY5 $\left(\mathrm{CY} 5_{i j}\right)$, which correspond to the copy numbers of objective and control samples, respectively, at the $i$ th BAC clone in the $j$ th objective sample. The $\log$ fluorescence ratio $x_{i j}$ is calculated as

$$
x_{i j}=\log _{2}\left(\mathrm{CY} 3_{i j} / \mathrm{CY} 5_{i j}\right) .
$$

Note that Eq. (1) is conceptual but not necessarily precise because it always requires correction. Various correction methods are available for various artifacts involved in microarray measurements. The most popular one is to introduce the correction term:

$$
f_{j}\left(\log _{2} \mathrm{CY} 3_{i j}+\log _{2} \mathrm{CY} 5_{i j}\right),
$$

which is a function of total fluorescence intensity.

Consequently, the objective of our method can be described as to estimate the mean local aberration $Z_{i j}$ from the observed log fluorescence ratio $x_{i j}$.

\section{Comb Model}

\subsection{The Simplest Comb Model}

Provided that every objective sample consists of homogeneous cells, let $N_{i j}$ and $N_{0}$ denote the DNA copy number of a clone $i$ in an objective sample $j$ and that of a control sample, respectively. As the control sample, we prepared normal cells that are all diploid DNA, i.e., $N_{0}=2$. 
We obtain the log fluorescence ratio $x$ in a similar fashion to that in the gene expression measurement:

$$
\begin{aligned}
x_{i j} & =\log _{2} \frac{c_{j} N_{i j}}{c_{0} N_{0}}+\nu_{i}+\mu_{j}^{\prime}+\epsilon_{i j} \\
& =\log _{2}\left(N_{\mathrm{P} j}+N_{\mathrm{C} i j}\right)+\nu_{i} \\
& +\mu_{j}^{\prime}+\log _{2}\left(c_{j} / c_{0} N_{0}\right)+\epsilon_{i j}, \\
& =\log _{2}\left(\frac{1}{N_{\mathrm{P} j}} N_{\mathrm{C} i j}+1\right)+\nu_{i}+\mu_{j}+\epsilon_{i j},
\end{aligned}
$$

where $\nu_{i}$ and $\mu_{j}^{\prime}$ are constant biases that denote mean log fluorescence ratios of the BAC clone $i$ and the sample $j$, respectively. $c_{j}$ and $c_{0}$ are constant factors proportional to the number of cells in the corresponding sample, hybridization efficiency, and so on, corresponding to the $j$-th objective sample and the control sample, respectively. The unknown factors, $c_{j}, c_{0}$, are united to a single bias term, $\mu_{j} \equiv \mu_{j}^{\prime}+\log _{2}\left(c_{j} N_{\mathrm{P} j} / c_{0} N_{0}\right) . \quad \epsilon_{i j}$ denotes a residual component that is assumed to obey a normal distribution with mean 0 and variance $\sigma^{2}$. The variance $\sigma^{2}$ corresponds to observation error and its value is assumed to be known before our analysis.

In the following discussion, we ignore the clone-wise bias $\nu_{i}$, because it is corrected by some common methods, such as Eq. (2). We also omit $j$, because our method deals with individual samples. Consequently, Eq. (3) becomes simply:

$$
x_{i}=\log _{2}\left(\frac{1}{N_{\mathrm{P}}} N_{\mathrm{C} i}+1\right)+\mu+\epsilon_{i} .
$$

Thus, when the variance $\sigma^{2}$ is small enough, the expected local aberration $i$ can be approximately obtained as:

$$
Z_{i}=E\left[N_{\mathrm{C} i}\right] \approx N_{\mathrm{P}}\left(2^{x_{i}-\mu}-1\right) .
$$

The unknown bias $\mu$ cannot be ignored, because it involves many aspects of variability in microarray slides, such as the inequality between the total amounts of DNA in objective and control samples and the asymmetry in the fluorescence of CY3 and CY5.

\subsection{Comb Fitting Based on the Sim- plest Comb Model}

Given the observed fluorescence ratio $X=$ $\left(x_{1}, \cdots, x_{i}, \cdots\right)$ for a sample, the likelihood of the unknown parameter $\theta=\{\mu\}$ is defined as

$$
\begin{gathered}
L(\theta \mid X)=\prod_{i} \prod_{k \in K} p\left(x_{i} \mid N_{\mathrm{C} i}=k, \mu\right) \\
P\left(N_{\mathrm{C} i}=k\right), \\
p\left(x_{i} \mid N_{\mathrm{C} i}=k, \mu\right)=\frac{1}{\sqrt{2 \pi \sigma^{2}}} \\
\exp \left[-\frac{1}{2 \sigma^{2}}\left(x_{i}-m_{k}\right)^{2}\right]
\end{gathered}
$$

where we assume that the residual $\epsilon_{i}$ is an independent normal noise with variance $\sigma^{2}, \theta$ represents the unknown parameter $\mu, K=$ $\left\{-N_{\mathrm{P}}, \cdots,-1,0,1,2, \cdots\right\}$ is a set of possible values of local aberrations, and $m_{k}$ denotes the Gaussian center defined by

$$
m_{k}=\log _{2}\left(\frac{1}{N_{\mathrm{P}}} k+1\right)+\mu .
$$

$p\left(N_{\mathrm{C}_{i}}=k\right)$ is the a priori probability, in short 'prior', of local aberration being $k$ at the $i$ th clone. In the simplest method, it is set to be equal for all $k$, but we discuss some advanced ways to incorporate a priori knowledge in Section 5.2.

We can determine $\mu$ to maximize the likelihood $L(\theta \mid X)$. For the mixture of normal distributions, their Gaussian centers $m_{k}$ are aligned in a pre-determined manner and shifted by a single location parameter $\mu$. In the following, we call this mixture model a comb model and call $m_{k}$ the $k$ th comb tooth. This maximum likelihood estimation corresponds to fitting the comb model into the data distribution by shifting the location $\mu$ of the comb. Figure 1 shows the concept of this comb fitting.

Since the comb teeth, $\log _{2}\left(N_{\mathrm{P}}+k\right)+\mu$, have non-equal intervals, there is a single location at which the set of comb teeth fits best into an ideal data set that obeys a normal mixture

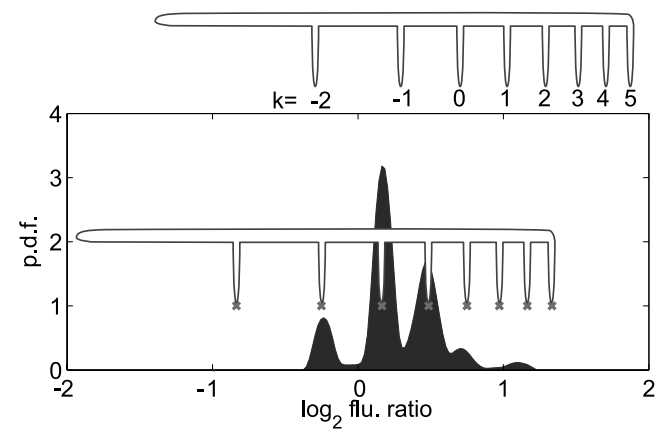

Fig. 1 Conceptual diagram of comb fitting. The comb, whose teeth are aligned at certain intervals, is fitted into the distribution of $\log$ fluorescence ratios. The mean local aberration $Z$ is then obtained. 
distribution with Gaussian centers, which have non-equal intervals, and a homogeneous Gaussian variance $\sigma^{2}$.

\subsection{Contamination by Normal Cells}

Let $b$ denote the contamination rate of normal somatic cells whose copy number is $N_{0}=2$ at all clones and assume that the ploidy number $N_{\mathrm{P}}$ of the tumor cells in the objective sample is homogeneous. When the contamination level is not negligible, the fluorescence ratio $x_{i j}$ becomes

$$
\begin{aligned}
x_{i j}= & \log _{2} \frac{c_{j}}{c_{0}} \frac{b_{j} N_{0}+\left(1-b_{j}\right) N_{i j}}{N_{0}} \\
& +\mu_{j}^{\prime}+\nu_{i}+\epsilon_{i j}
\end{aligned}
$$

which leads to a modification of the comb teeth into

$$
\begin{aligned}
& m_{k}=\log _{2}\{B k+1\}+\mu, \\
& B_{j}=\left(\frac{b_{j}}{1-b_{j}} N_{0}+N_{\mathrm{P} j}\right)^{-1} .
\end{aligned}
$$

We can easily find that when $b_{j}=0$, i.e. $B_{j}=$ $N_{\mathrm{P}}{ }_{j}^{-1}$, it is equivalent to the simplest case (3). In addition, consider the extreme case of highlevel contamination, $b_{j} \rightarrow 1$, i.e., $B_{j} \rightarrow 0$; in this case, the comb teeth become insensitive to the local aberration $k$.

The two unknown parameters $b_{j}$ and $N_{\mathrm{P} j}$ are united into $B_{j}$, and if $B_{j}$ is obtained the expected local aberration is obtained as

$$
Z_{i}=E\left[N_{\mathrm{C} i}\right] \approx B_{j}^{-1}\left(2^{x_{i}-\mu}-1\right) .
$$

Thus, we estimate $\theta=\{B, \mu\}$ by the maximum likelihood estimation instead of considering $b$ and $N_{\mathrm{P}}$.

\subsection{Heterogeneity in Ploidy Number}

Assume that there is considerable heterogeneity in ploidy number $N_{\mathrm{P}}$ of objective tumor cells; the ratios of $N_{\mathrm{P}}=2, N_{\mathrm{P}}=3, \cdots$ are given by $\beta^{(2)}, \beta^{(3)}, \cdots$, respectively, with the condition $\sum_{N_{\mathrm{P}}} \beta^{\left(N_{\mathrm{P}}\right)}=1$. We assume that the contamination ratio $b$ of normal cells is also considerable, but the local aberration $N_{\mathrm{C}}$ is the same for all cells regardless of their ploidy number.

In this case, comb teeth take precisely the same form as Eq. (10), except that the definition of the parameter $B_{j}$ is

$$
B_{j}=\left(\frac{b_{j}}{1-b_{j}} N_{0}+\sum_{N_{\mathrm{P}}} \beta^{\left(N_{\mathrm{P}}\right)} N_{\mathrm{P}}\right)^{-1}
$$

Consequently, comb fitting needs only two parameters, $\mu$ and $B$, even when there is het- erogeneity in the ploidy number $N_{\mathrm{P}}$.

Even if there is considerable heterogeneity in local aberrations for some clones, it is negligible when its amount is small relative to the number of total clones in the sample.

Accordingly, the problem becomes obtaining only two parameters, $\mu$ and $B$, in all of the above cases. The most ideal case, which is considered first, is equivalent to assuming $B=0$ in Eq. (10). How to determine the parameters will be described in the next section.

\section{Maximum Likelihood Estimation and Its Problems}

Maximum likelihood estimation estimates $\theta=\{\mu, B\}$ to maximize the log likelihood function $L(\theta \mid X)$.

Figure 2 shows a contour plot of the functional relationship of the log likelihood with the parameters $\mu$ and $B$ when applied to typical array CGH profile data. The mark ' $\mathrm{x}$ ' in the contour denotes the maximum likelihood solution, $\left(\mu_{\mathrm{MAX}}, B_{\mathrm{MAX}}\right)$. Obvious multi-modality can be seen in the log likelihood landscape; such multi-modality is often observed, especially when the heterogeneity is low. To obtain the maximum likelihood solution, we used a mesh search method, which searches the mesh over the space of $\mu$ and $B$ for the maximum point. This was done because simple gradientbased methods fail to obtain the optimal solution due to the multi-modality.

There are two reasons causing such multimodality to appear. The first is that a steppingstone-like alternative, whose comb teeth correspond to the first, third and fifth teeth of the optimal comb for example, may have a comparable likelihood. The second is that as $B$ be-

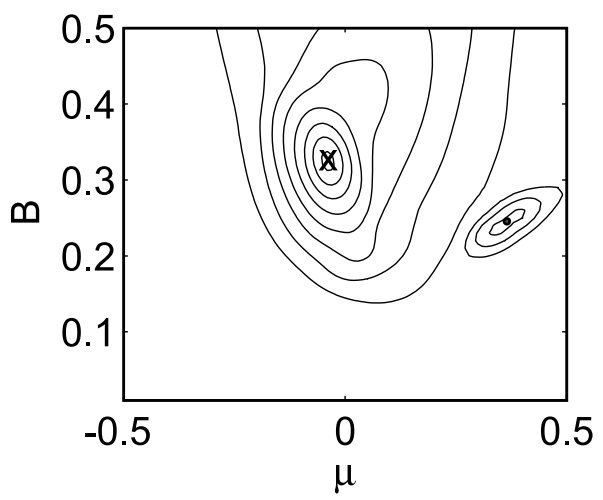

Fig. 2 Contour plot of log-likelihood. The mark ' $x$ ' in the contour denotes the maximum likelihood solution. 


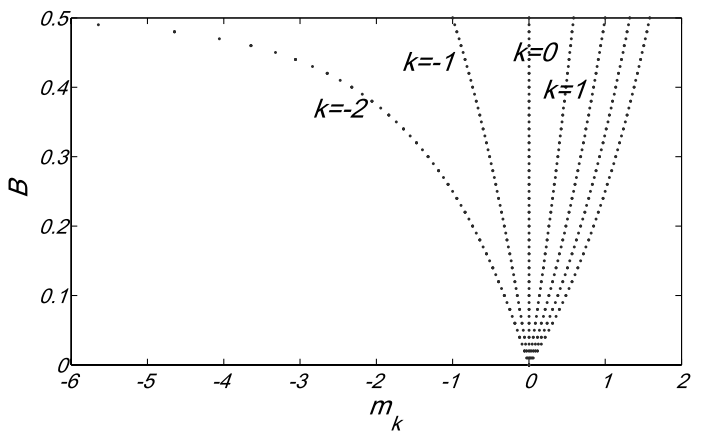

Fig. 3 Comb tooth when $B$ is small.

comes small, the intervals between teeth cannot be distinguished, which makes the likelihood invariant with respect to the shift in the comb position.

Figure 3 shows the relationship between $B$ values and comb tooth intervals. For a small $B$, tooth intervals are narrow and the difference between neighboring intervals becomes small. When the neighboring intervals are similar, unit shift of the comb does not lead to a significant difference in fitting performance, which gives the solution ambiguity. There is another difficulty. Because the residual variance $\sigma^{2}$ is fixed, narrower tooth intervals lead to larger overlap between two adjacent Gaussian distributions, which makes it difficult to distinguish them. Accordingly, when $B$ approaches 0 , the simple maximum likelihood method to perform comb fitting becomes difficult.

A small $B$ means a high contamination rate. To overcome the difficulties due to high contamination rate, we propose several devices, such as designing data preprocessing, introducing a priori knowledge, or brute force by hand-tuning. We explain these devices in the next section.

\section{Modifications of Comb Fitting}

\subsection{Preprocessing}

We applied spatial filtering on each chromosome as a data preprocessing device. Two types of one-dimensional spatial filters, lowess and block filter, were tried. The lowess filter is based on the assumption that log fluorescence ratio varies continuously along the locus coordinate in a single chromosome. The block filter assumes three block regions in a chromosome, where the copy number is assumed to be identical in a single block. Although there are many segmental or other filtering procedures $^{2) \sim 5), 7) \sim 9), 12), 13)}$, we did not tried all of them because we only need a filtering process
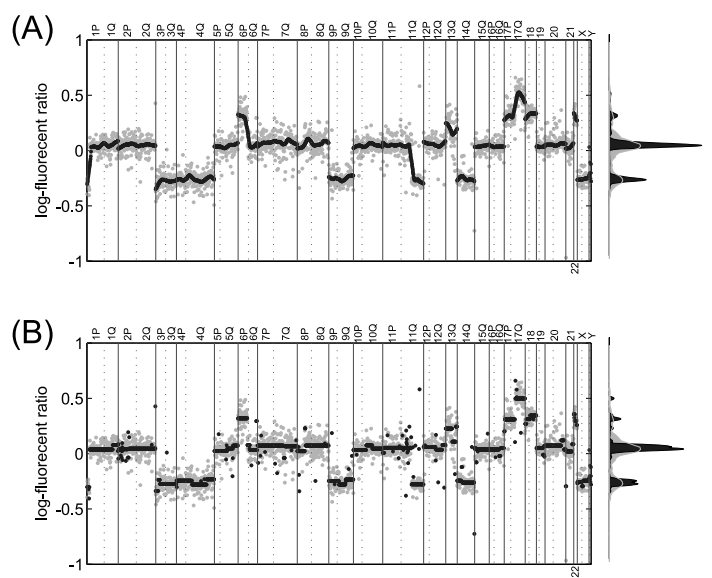

Fig. 4 Smoothing filters as data preprocessing. Gray and black spots denote original and filtered data, respectively. The Gray and black histograms on the right hand side are corresponding to the original and filtered data, respectively.

in order to obtain a better global profile for the histogram of the log fluorescence ratio, and the various types of filtering do not yield large differences in the histogram.

Figures $4(\mathrm{~A})$ and $4(\mathrm{~B})$ show the results after applying the lowess filter and the block filter, respectively. With either filter, the comb tooth structure was clarified, as can be seen in the histogram shown to the right of the corresponding panel. Note that the final results of the comb fitting are comparable despite which filter is used because the two histograms are similar.

DNA copy number aberrations sometimes seem to have a block structure, i.e., a certain region of a $\mathrm{P}$-arm or Q-arm exhibits a fixed copy number gain or loss. To obtain such a block structure, the block filter splits the chromosome into three blocks, each with a unique copy number. To determine the break points of the three blocks, we define the distortion measure based on the comb:

$$
D_{r}=\sum_{i \in \text { chromosome } r}\left(x_{i}-m(i)\right)^{2},
$$

where $r$ denotes chromosome index and $m(i)$ denotes mean log fluorescence ratio of the block to which the $i$ th clone belongs. Block filtering determines two break points for each chromosome $r$ to minimize $D_{r}$. Pre-defined outlier clones are omitted from the filtering.

\subsection{Using a priori Knowledge}

If we have biological knowledge about copy 
number aberrations, it can be used as a priori knowledge for improving the estimation made by comb fitting.

Most local aberrations of chromosomes are at most a single gain or loss. If comb fitting suggests that a large area of a chromosome in diploid sample cells has lost its two copies, this result is not natural, because such a loss would cause serious damage even to tumor cells. Because our comb model is formulated as a probabilistic model, such a priori knowledge can be incorporated as the prior distribution. The prior $p\left(N_{\mathrm{C} i}=k\right)$ represents the probability that a local aberration of the $i$ th clone is $k$. There are three possible ways of preparing the prior: subjective tuning, empirical tuning and recursive tuning.

Subjective tuning is based on a subjective belief about the frequency of copy number aberrations. As a standard setting for diploid tumor cells, we used the following prior:

$$
\begin{aligned}
& p\left(N_{\mathrm{C}}=-2\right)=\varepsilon C \\
& p\left(N_{\mathrm{C}}=-1\right)=5 C \\
& p\left(N_{\mathrm{C}}=0\right)=10 C \\
& p\left(N_{\mathrm{C}}=1\right)=2 C \\
& p\left(N_{\mathrm{C}}=2\right)=C \\
& p\left(N_{\mathrm{C}}=3\right)=C,
\end{aligned}
$$

where $\varepsilon$ is a small number $(=0.01)$ that represents the rareness of the event $N_{\mathrm{C}}=-2$; however, $\varepsilon=0$ incurs too large a penalty in the case that $Z$ becomes -2 , possibly due to occasional noise. $C$ is set from the normalization condition $\sum_{k=-2}^{3} p\left(N_{\mathrm{C}}=k\right)=1$.

When much information is available about the occurrence rates of local aberrations, empirical tuning is advantageous. Namely, we set the prior probability $p\left(N_{\mathrm{C}}=k\right)$ directly to the empirical ratio of copy numbers $N_{\mathrm{C}}=$ $-2,-1,0,1,2,3, \cdots$. Note that setting $p\left(N_{\mathrm{C}}=\right.$ $k)=\varepsilon>0$ will be better even when $N_{\mathrm{C}}=k$ has not occurred empirically.

We may consider recursive tuning of the prior when we have insufficient background information but have a fairly large amount of array CGH data. Namely, the frequencies of copy number aberrations estimated using a subjectively tuned prior are used as new background information for the next empirical tuning.

When it is known, the dependence on ploidy and/or chromosome numbers should be used in each tuning method.

\subsection{Hand Tuning}

Either when $B$ is close to 0 or when there is significant heterogeneity of local aberrations in sample cells, our comb model with the abovementioned modifications has difficulty in obtaining an appropriate solution. In such a case, one possible way is hand tuning. In hand tuning, we need to set at most three pairs of reference values to determine the two unknown parameters $\mu$ and $B$ unequivocally.

For example, if we set log fluorescence ratios $x^{(-1)}, x^{(0)}, x^{(1)}$ to $Z=-1,0,1$, respectively, $\mu$ and $B$ are unequivocally determined and hence the remaining $x^{(-2)}, x^{(2)}, x^{(3)}, \cdots$ corresponding to $Z=-2,2,3, \cdots$ are determined automatically.

\section{A Case Study}

Figure 5 shows a demonstrative analysis of array CGH observation data obtained from a frozen sample of human neuroblastoma.

In neuroblastoma, it is known that chromosomal aberrations in the first and seventeenth chromosomes have a high correlation with the patient's prognosis. We conducted fluorescence in situ hybridization (FISH) observations on these two chromosomes and found the following points.

- The sample cells have three or four copies of both the first and seventeenth chromosomes.

- The copy number ratio between the Q-arm and the centromere of the seventeenth chromosome, $17 \mathrm{q} / 17 \mathrm{cen}$, is $8 / 3$ or $9 / 4$.

According to these observations, we conclude that this sample has heterogeneity in the ploidy number, which lies between triploid and tetraploid, and the local aberration of $17 \mathrm{q}$ is +5 gain.

In Fig. 5 (a), a gray point denotes original log fluorescence ratio observed at each clone, and a black point denotes the corrected value after block filtering. Figure 5 (b) shows the histograms of the original and the filtered log ratios, which are depicted by gray and black colors, respectively. Although the histogram of the original ratios shows a single large peak, that of the filtered ratios shows clear multi-modality, which seems to fit the comb model. Accordingly, we applied comb fitting to these blockfiltered data. We set the constant $\sigma^{2}$, the variance of each single comb tooth, at the mean variance over blocks extracted by the block filtering; namely, it is set at the mean squared 

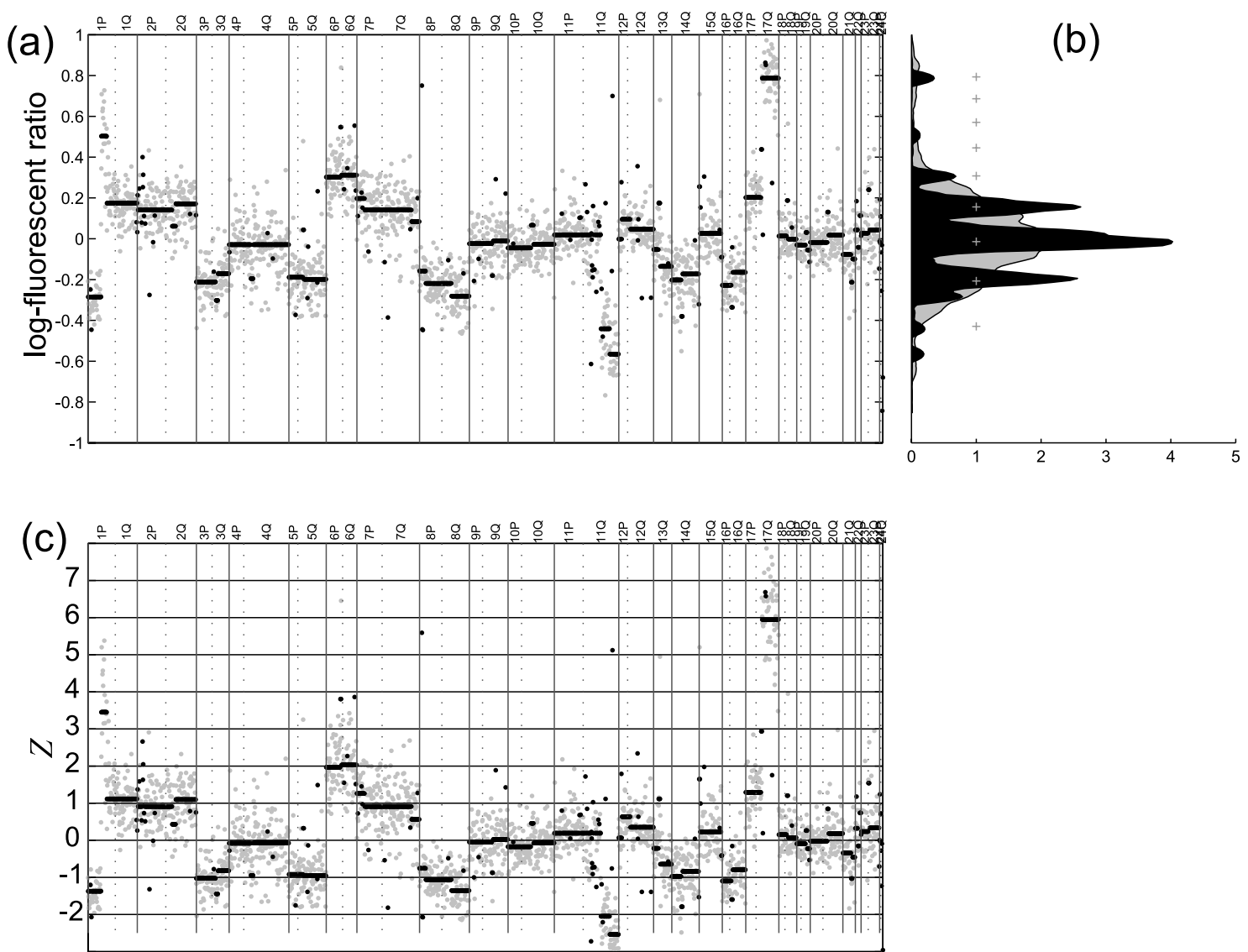

Fig. 5 Demonstration of comb fitting. Demonstration of comb fitting for a sample that has large and complex chromosomal aberrations.

residual of the block filtering divided by the mean number of clones within a block. Although it is possible in principle to estimate $\sigma^{2}$ as another parameter of the likelihood, we regarded it as a constant because increasing the number of parameters makes it difficult to estimate them against the multi-modality of the likelihood function. As the prior, we used a uniform distribution to reflect the lack of background knowledge.

The results of the comb fitting are shown by ' + ' marks in Fig. 5 (b), which denote comb teeth fitted into the histogram. Figure $5(\mathrm{c})$ shows the mean local aberration values, $Z$, obtained by comb fitting. The $Z$ value for each clone and its block-wise mean are plotted as gray and black spots, respectively. We can see that the $Z$ values, especially for the block-wise means, densely cluster around integer numbers.

The $Z$ values at the $\mathrm{Q}$-arm of the seventeenth chromosome, $17 \mathrm{q}$, cluster around the +6 gain, which differs by one copy from the FISH obser- vation ( +5 gain). Our result is consistent with the FISH data, however, if we assume that the baseline (0) of the $Z$ values corresponds to a single copy loss. Actually, the second peak of the log likelihood of the comb model corresponded to the alternative solution. Since the difference between their peak heights is small, we can probably obtain the second peak as the best solution if we use appropriate a priori knowledge.

We found in Figs. 5(a) and 5(c) that log fluorescence ratios and mean local aberrations of clones have noisy distribution centered at block-wise filtered values. Concerning variances of residuals, those in the log fluorescence ratio are almost homogeneous at any location (Fig. $5(\mathrm{a})$ ), while those in the mean local aberration are large because the mean local aberration itself is large (Fig. 5(c)). This is because the comb tooth intervals are narrow when the mean local aberration is large, and hence expansion from the log fluorescence measurement to the mean local aberration becomes large. 
Therefore, a large portion of the residual variation in copy number aberrations is due to observation noise in the log fluorescence ratio, rather than as an outcome of local genetic copy number aberration such as homozygous gains or losses.

\section{Discussion}

Appropriate application of comb fitting often requires subjective setting of parameters based on a priori knowledge. Although this may seem, at first glance, to violate the objectivity of data analysis, it is objective enough because the a priori knowledge must be expressed explicitly as the prior distribution to meet the probabilistic estimation process with the comb model.

From the Bayesian point of view, data analysis of all sorts includes inevitable bias from a researcher's subjective a priori beliefs about the analysis targets. Therefore, what is important for sound data analysis is to explicitly express $a$ priori knowledge in the form of prior probability. The subjective tuning and hand tuning of the comb fitting, discussed in Section 5.3, were based on such an idea.

When the prior probability is available, we can update it by using a posteriori knowledge obtained from the observed data, which enhances the objectivity of the analysis. The empirical tuning and recursive tuning of the prior, discussed in Section 5.2, were based on this idea.

\section{Conclusion}

We have developed a method called comb fitting, which determines the copy number of DNA corresponding to each BAC clone from each fluorescence ratio measured by array CGH.

Automatic comb fitting can be used even when there is considerable contamination by normal cells or when tumor cells have heterogeneous ploidy numbers. We also proposed modifications using a priori knowledge and/or hand tuning, which help comb fitting when automatic fitting is difficult to apply, as in cases where large contamination of normal cells or large heterogeneity in local aberrations exists.

Acknowledgments The authors are grateful to Dr. Burt G Feuerstein, Dr. Donna Albertson and Dr. Dan Pinkel (Cancer Center and Department of Laboratory Medicine, University of California) for array CGH data acquisition, to Dr. Jane Fridlyand (Cancer Center and Department of Laboratory Medicine, University of California) for fluorescence data prepocessing, to Dr. Yasuhiko Kaneko (Division of Chemotherapy, Saitama Cancer Center Research Institute) for FISH analysis of a demonstration sample, and to Dr. Akira Nakagawara (Chiba Cancer Center Research Institute) for discussions on characteristics of neuroblastoma samples.

This work was supported by KAKENHI (Grant-in-Aid for Scientific Research) on Priority Areas "Applied Genomics" from the Ministry of Education, Culture, Sports, Science and Technology of Japan.

\section{References}

1) Barrett, M.T., Scheffer, A., Ben-Dor, A., Sampas, N., Lipson, D., Kincaid, R., Tsang, P., Curry, B., Baird, K., Meltzer, P.S., Yakhini, Z., Bruhn, L. and Laderman, S.: Comparative genomic hybridization using oligonucleotide microarrays and total genomic DNA, Proc. Natl. Acad.Sci.USA, Vol.101, No.51, pp.17765-17770 (2004).

2) Carvalho, B., Ouwerkerk, E., Meijer, G.A. and Ylstra, B.: High resolution microarray comparative genomic hybridisation analysis using spotted oligonucleotides, J. Clin. Pathol., Vol.57, No.6, pp.644-646 (2004). Evaluation Studies.

3) Daruwala, R.S., Rudra, A., Ostrer, H., Lucito, R., Wigler, M. and Mishra, B.: A versatile statistical analysis algorithm to detect genome copy number variation, Proc. Natl. Acad. Sci. USA, Vol.101, No.46, pp.16292-16297 (2004).

4) Eilers, P.H.C. and de Menezes, R.X.: Quantile smoothing of array CGH data, Bioinformatics, Vol.21, No.7, pp.1146-1153 (2005). Evaluation Studies.

5) Fridlyand, J., Snijders, A.M., Pinkel, D., Albertson, D.G. and Jain, A.N.: Hidden Markov models approach to the analysis of array CGH data, Journal of Multivariate Analysis, Vol.90, No.1, pp.132-153 (2004).

6) Hodgson, G., Hager, J.H., Hariono, S., Wernick, M., Moore, D., Albertson, D.G., Pinkel, D., Collins, C., Hanahan, D. and Gray, J.W.: Genome scanning with array CGH delineates regional alterations in mouse islet carcinomas, Nature Genetics, Vol.29, pp.459-464 (2001).

7) Hsu, L., Self, S.G., Grove, D., Randolph, T., Wang, K., Delrow, J.J., Loo, L. and Porter, P.: Denoising array-based comparative genomic hybridization data using wavelets, Biostatistics, Vol.6, No.2, pp.211-226 (2005). 
8) Hupe, P., Stransky, N., Thiery, J.-P., Radvanyi, F. and Barillot, E.: Analysis of array CGH data: from signal ratio to gain and loss of DNA regions, Bioinformatics, Vol.20, No.18, pp.3413-3422 (2004). Evaluation Studies.

9) Jong, K., Marchiori, E., Meijer, G., Vaart, A.V.D. and Ylstra, B.: Breakpoint identification and smoothing of array comparative genomic hybridization data, Bioinformatics, Vol.20, No.18, pp.3636-3637 (2004). Evaluation Studies.

10) Lai, W.R., Johnson, M.D., Kucherlapati, R. and Park, P.J.: Comparative analysis of algorithms for identifying amplifications and deletions in array CGH data, Bioinformatics, Vol.21, No.19, pp.3763-3770 (2005).

11) Lucito, R., Healy, J., Alexander, J., Reiner, A., Esposito, D., Chi, M., Rodgers, L., Brady, A., Sebat, J., Troge, J., West, J.A., Rostan, S., Nguyen, K.C.Q., Powers, S., Ye, K.Q., Olshen, A., Venkatraman, E., Norton, L. and Wigler, M.: Representational oligonucleotide microarray analysis: A high-resolution method to detect genome copy number variation, Genome Res., Vol.13, No.10, pp.2291-2305 (2003).

12) Myers, C.L., Dunham, M.J., Kung, S.Y. and Troyanskaya, O.G.: Accurate detection of aneuploidies in array CGH and gene expression microarray data, Bioinformatics, Vol.20, No.18, pp.3533-3543 (2004). Evaluation Studies.

13) Picard, F., Robin, S., Lavielle, M., Vaisse, C. and Daudin, J.-J.: A statistical approach for array CGH data analysis, BMC Bioinformatics, Vol.6, No.1, p.27 (2005).

14) Pinkel, D., Segraves, R., Sudar, D., Clark, S., Poole, I., Kowbel, D., Collins, C., Kuo, W.-L., Chen, C., Zhai, Y., Dairkee, S.H., Ljung, B.M. and Gray, J.W.: High resolution analysis of DNA copy number variation using comparative geneomic hybridization to microarrays, Nature Genetics, Vol.20, pp.207-211 (1998).

15) Pollack, J.R., Sorlie, T., Perou, C.M., Rees, C.A., Jeffrey, S.S., Lonning, P.E., Tibshirani, R., Botstein, D., Borresen-Dale, A. and Brown, P.O.: Microarray analysis reveals a major direct role of DNA copy number alteration in the transcriptional program of human breast tumors, Proc. Natl. Acad. Sci. USA, Vol.99, pp.12963-12968 (2002).

16) Pollack, J.R., Perou, C.M., Alizadeh, A.A., Eisen, M.B., Pergamenschikov, A., Williams, C.F., Jeffrey, S.S., Botstein, D. and Brown, P.O.: Genome-wide analysis of DNA copynumber changes using cDNA microarrays, $\mathrm{Na}$ ture Genetics, Vol.23, pp.41-46 (1999).

17) Snijders, A.M., Nowak, N., Segraves, R., Blackwood, S., Brown, N., Conroy, J., Hamil- ton, G., Hindle, A.K., Huey, B., Kimura, K., Law, S., Myambo, K., Palmer, J., Ylstra, B., Yue, J.P., Gray, J.W., Jain, A.N., Pinkel, D. and Albertson, D.G.: Assembly of microarrays for genome-wide measurement of DNA copy number, Nat. Genet., Vol.29, No.3, pp.263-264 (2001).

18) Veltman, J.A., Fridlyand, J., Pejavar, S., Olshen, A.B., Korkola, J.E., DeVries, S., Carroll, P., Kuo, W.-L., Pinkel, D., Albertson, D., Cordon-Cardo, C., Jain, A.N. and Waldman, F.M.: Array-based Comparative Genomic Hybridization for Genome-Wide Screening of DNA Copy Number in Bladder Tumors, Cancer Research, Vol.63, pp.2872-2880 (2003).

19) Willenbrock, H. and Fridlyand, J.: A comparison study: applying segmentation to array CGH data for downstream analyses, Bioinformatics, Vol.21, No.22, pp.4084-4091 (2005).

(Received July 31, 2006)

(Accepted September 11, 2006)

(Released November 29, 2006)

(Communicated by Susumu Goto)

(Paper version of this article can be found in the IPSJ Transactions on Bioinformatics, Vol.47 No.SIG17(TBIO1), pp.73-82.)

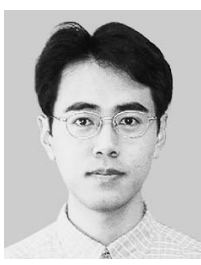

Shigeyuki Oba received his M.S. degree from Kyoto University in 1998, M.S. and Ph.D. degrees from Graduate School of Information Science in Nara Institute of Science and Technology in 2001 and 2002, respectively. He has been in Nara Institute of Science Technology, since 2002 as a researcher, and since 2003 as an assistant professor. His current research interests are machine learning and their application to bioinformatics.

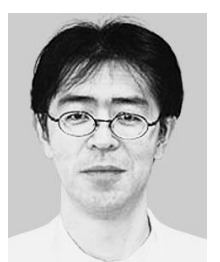

Nobumoto Tomioka received his M.D. degree from Hokkaido University in 1991. He started research in medical genomics in Saitama and Chiba cancer center from 1999, then worked on array CGH for neuroblastoma in UCSF from 2001 to 2004. He is currently working at the 1st Department of Surgery, Hokkaido University, Graduate School of Medicine as an assistant instructor. 


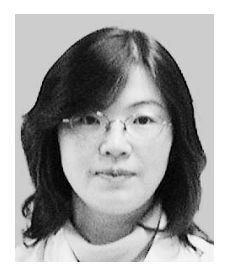

Miki Ohira received M.S. and Ph.D. degrees from the University of Tokyo in 1991 and 1997, respectively. After getting M.S. degree, she had been in Kazusa DNA Research Institute as a research scientist, and then joined the Chiba Cancer Center Research Institute in 1998 working in medical genomics as a staff researcher. She was co-recipient of the Luca Lotti Award of the Advances in Neuroblastoma Research in 2004. Her current research interests include genetics of neuroblastoma development and constructing a DNA chip baseddiagnostic system of pediatric cancers.

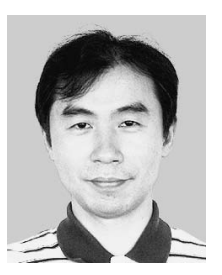

Shin Ishii was born in 1962. He received his B.E., M.E. and Ph.D. degrees from the University of Tokyo in 1986, 1988 and 1997, respectively. He had worked in Ricoh Co. Ltd. and ATR Human Information Research Laboratories since 1988 and 1994, respectively. Since 1997 he had been in Nara Institute of Science and Technology as an associate professor and since 2001 as a professor with Graduate School of Information Science. His current research interests are statistical bioinformatics, systems biology, computational neuroscience and artificial intelligence. He is a member of IPSJ, IEICE, JNNS and Biophysical Society. 